\title{
NUMERICAL COMPUTATION FOR THE NON-CUTOFF RADIALLY SYMMETRIC HOMOGENEOUS BOLTZMANN EQUATION
}

\author{
LÉo GLAngetas, IBrahim JRAD \\ Université de Rouen Normandie, UMR 6085-CNRS, Mathématiques \\ Avenue de l'Université, BP.12, 76801 Saint Etienne du Rouvray, France
}

\begin{abstract}
For the non cutoff radially symmetric homogeneous Boltzmann equation with Maxwellian molecules, we give the numerical solutions using symbolic manipulations and spectral decomposition of Hermit functions. The initial data can belong to some measure space.
\end{abstract}

\section{Contents}

1. Introduction 2

1.1. The Boltzmann equation 2

1.2. Results on the Boltzmann equation 3

2. Theoretical results 4

2.1. Linearization of the Boltzmann equation 4

2.2. The spectral problem 5

3. Numerical computations 9

3.1. Computation of the eigenvalues 10

3.2. Computation of the nonlinear eigenvalues 11

3.3. Numerical solutions of the linear problem 12

3.4. Numerical solutions of the non-linear part 13

3.5. Discussions on the symbolic computation 14

4. Radial bi-Gaussian initial value 16

5. Numerical results for initial measure data 18

6. Conclusion 20

Acknowledgments 22

7. Appendix 22

7.1. Rescaling of the solution 22

7.2. Measure initial data 23

7.3. Some results on the spherical harmonics 25

References 25

Date: October 14, 2018.

2010 Mathematics Subject Classification. 34K08, 35Q20, 35-04, 35P05, 35P30, $80 \mathrm{M} 22$.

Key words and phrases. Boltzmann equation, kinetic equations, spectral decomposition, symbolic computation, numerical computation.

The second author is supported by a grant from Lebanon. 


\section{INTRODUCTION}

1.1. The Boltzmann equation. The Boltzmann equation, derived by Boltzmann in 1872 (and Maxwell 1866), models the behavior of a dilute gas (see [8]). As we know, Boltzmann has created a theory which described the movement of gases as balls which could bump and rebound against each other [11, 19]. This model can be considered by one of many cases which represent the so-called kinetic equation. Presently, the diversity of sciences and applications contains these models such as rarefied gas dynamics, semiconductor modeling, radiative transfer, and biological and social sciences. This type of equations is made by including a combination of a linear transport term and several interaction terms which provide the time evolution of the distribution of particles in the phase space. The equation that bears his name is the following

$$
\partial_{t} f+v \cdot \nabla_{x} f=\mathbf{Q}(f, f)
$$

where $f=f(t, x, v) \geq 0$ is the probability density to find a particle at the time $t$, on the position $x$ and with velocity $v$ where the physical and the velocity space are located in three dimensions. The term $v \cdot \nabla_{x} f$ describes the free action of particles and $\mathbf{Q}(f, f)$ is a bilinear operator which describes the binary collision process. It is called the Boltzmann collision operator and given by

$$
\mathbf{Q}(g, f)(v)=\int_{\mathbb{R}^{3}} \int_{\mathbb{S}^{2}} B\left(v-v_{*}, \sigma\right)\left(g\left(v_{*}^{\prime}\right) f\left(v^{\prime}\right)-g\left(v_{*}\right) f(v)\right) d v_{*} d \sigma
$$

where for $\sigma \in \mathbb{S}^{2}$, the symbols $v_{*}^{\prime}$ and $v^{\prime}$ are abbreviations for the expressions,

$$
v^{\prime}=\frac{v+v_{*}}{2}+\frac{\left|v-v_{*}\right|}{2} \sigma, \quad v_{*}^{\prime}=\frac{v+v_{*}}{2}-\frac{\left|v-v_{*}\right|}{2} \sigma,
$$

which are obtained in such a way that collision preserves momentum and kinetic energy, namely

$$
v_{*}^{\prime}+v^{\prime}=v+v_{*}, \quad\left|v_{*}^{\prime}\right|^{2}+\left|v^{\prime}\right|^{2}=|v|^{2}+\left|v_{*}\right|^{2}
$$

where $|\cdot|$ is the Euclidean norm on $\mathbb{R}^{3}$. Note that $v, v^{\prime}$ are the velocities before collision and $v_{*}, v_{*}^{\prime}$ the velocities after collision.

The non-negative cross section $B(z, \sigma)$ depends only on $|z|$ and the scalar product $\frac{z}{|z|} \cdot \sigma=\cos \theta$ where $\theta$ is the deviation angle. Without loss of generality, we may assume that this cross section is supported on the set $\cos \theta \geq 0$. See for instance [30] for more details on the cross section and [38] for a general collision kernel. For physical models, it usually takes the form

$$
B\left(v-v_{*}, \sigma\right)=\Phi\left(\left|v-v_{*}\right|\right) b(\cos \theta), \cos \theta=\frac{v-v_{*}}{\left|v-v_{*}\right|} \cdot \sigma, 0 \leq \theta \leq \frac{\pi}{2},
$$

where $\Phi\left(\left|v-v_{*}\right|\right)=\left|v-v_{*}\right|^{\gamma}$ is a kinetic factor and $\gamma>-3$. 
In this work, we consider the spatially homogeneous case, that means the density distribution $f=f(t, v)$ depends on the variables $t \geq 0$, $v \in \mathbb{R}^{3}$ and is uniform with respect to $x$. So that the Boltzmann equation reads as

$$
\left\{\begin{array}{l}
\partial_{t} f=\mathbf{Q}(f, f) \\
f(0, v)=F(v)
\end{array}\right.
$$

where the initial data $F$ is depends only on $v$. For the collision kernel, we study only the Maxwellian molecules and non-cutoff cases (see [13, $12,14,15,16,24,31])$, that means the kinetic factor $\Phi \equiv 1$ and

$$
b(\cos \theta) \approx \frac{1}{|\theta|^{2+2 s}}, \quad 0<s<1, \theta \in\left(0, \frac{\pi}{2}\right] .
$$

1.2. Results on the Boltzmann equation. With the previous assumption (the non-cutoff case) on the cross-section, there is existence of a weak solution for the Boltzmann equation (1.1) for a positive initial value $F \in L_{2+\delta}^{1}\left(\mathbb{R}^{3}\right)$. See [37] and many others.

Moreover, it is well-known that there is a regularization effect in Sobolev and Schwartz or analytic spaces for any time $t>0$ (we refer the reader to $[13,12]$ and recently [1]) and that the solutions converge to the Gaussian when the time tends to infinity ([24]).

An important point that our distribution lives in a multidimensional space: this reason make us think that we have a numerical problem because in this case the computational cost is more or less forbidden [19]. The study of the numerical part for kinetic equations is not obvious due to many difficulties come from the computational cost. To clarify more, we mention two of these difficulties: It is clear the appearing of multiple scales, and then to get out of the resolution of the stiff dynamics, one should build suitable numerical schemes [25, 26, 2, 17, 27, 18]. The other one is that the collision operator is defined by multidimensional integrals and to compute one should solve it point by point as physical space [35, 21]. To treat kinetic equations numerically, there is several ways which are used over the centuries until now: probabilistic numerical methods such as Direct Simulation Monte Carlo (DSMC) schemes [11,3], and, deterministic numerical methods such as finite volume, semi-Lagrangian and spectral schemes [19].

There are two important deterministic methods which are used in the past decades : the discrete velocity method (DVM) [23, 36, 6, 9, 33, 10] and the Fourier spectral method (FSM) [7, 34, 35, 22, 10]. Due to its discrete nature, the DVM preserves positivity of the distribution function, the $\mathrm{H}$-theorem and the exact conservation of mass, energy and momentum. Note that the Fourier spectral method is based on two main things : the truncation of the collision operator and the restriction of the distribution function to an appropriate cube, for more details see [35, 32]. 
Our goal is to present an alternative method to solve formally and numerically the homogeneous Boltzmann equation in the non-cutoff case. In this work, we consider the radial symmetric case and we use a spectral method : we first compute the spectral coefficients of the solution with a formal computation software (Maple ${ }^{\circledR} 13$; the codes can be provided). We then approximate these exact solutions and check the numerical results.

The used method helps us to motivate our work in several ways: It let us in the physical view understand more the behavior of the solutions and as we compute the first exact projections of the solutions on the spectral basis, that is in the numerical view, some other algorithms can be tested in the non-cutoff case (recall that the explicit 2D "BKW" solutions, obtained independently in [4, 28] are used to test the accuracy of the numerical methods in the case of a regular collision kernel $B \equiv 1$, see for example [10]). Finally, we do hope that our work will give some clues to formulate new mathematical conjectures.

The paper is organized as follows. In section 2, we state the main theoretical results. The numerical details and algorithms are provided in section 3. Sections 4 and 5 present the numerical results of the Boltzmann equation with different initial data for the Cauchy problem: we discuss in section 4 the results for a small $L^{2}$ initial data (bi-Gaussian); in section 5, we consider the case of a measure initial data. After that, we give a conclusion for this work. The paper ends with an appendix where we set some technical results.

\section{Theoretical Results}

In this section, we present some theoretical parts: we begin by linearizing the Boltzmann equation and giving the spectral decomposition of this equation.

2.1. Linearization of the Boltzmann equation. We remark that $Q(\mu, \mu)=0$ where the Gaussian function is defined by

$$
\mu(v)=\frac{1}{(2 \pi)^{3 / 2}} e^{-\frac{|v|^{2}}{2}}
$$

and it is a stationary solution of the Boltzmann equation. We consider now a perturbation $g$ of the Gaussian. Then the solution $f$ of (1.1) can be written as

$$
\begin{aligned}
& f(t, v)=\mu(v)+\sqrt{\mu(v)} g(t, v), \\
& F(v)=\mu(v)+\sqrt{\mu(v)} G(v) .
\end{aligned}
$$

It is easy to show that $g$ is a solution of the Cauchy problem

$$
\left\{\begin{array}{l}
\partial_{t} g+\mathcal{L}(g)=\Gamma(g, g) \\
\left.g\right|_{t=0}=g(0, v)=G(v)
\end{array}\right.
$$


where

$$
\mathcal{L}(g)=-\frac{1}{\sqrt{\mu}}[\mathbf{Q}(\sqrt{\mu} g, \mu)+\mathbf{Q}(\mu, \sqrt{\mu} g)]
$$

is a linear operator and

$$
\boldsymbol{\Gamma}(g, h)=\frac{1}{\sqrt{\mu}} \mathbf{Q}(\sqrt{\mu} g, \sqrt{\mu} h)
$$

is a nonlinear operator. We decompose the solution of (2.1) into a linear and nonlinear part:

$$
g(t, v)=\underbrace{e^{-t \mathcal{L}} G(v)}_{\text {linear part }}+\underbrace{e^{-t \mathcal{L}} h(t, v)}_{\text {nonlinear part }}
$$

where $e^{\alpha \mathcal{L}}$ is the exponential of the linear operator defined by his spectral decomposition (see below) and the new function $h(t, v)$ satisfies the following equation

$$
\left\{\begin{array}{l}
\partial_{t} h=e^{t \mathcal{L}} \boldsymbol{\Gamma}\left(e^{-t \mathcal{L}}(G+h), e^{-t \mathcal{L}}(G+h)\right), \\
h(0, v)=0 .
\end{array}\right.
$$

The linearized operator $\mathcal{L}$ is a positive unbounded symmetric operator on $L^{2}\left(\mathbb{R}_{v}^{3}\right)$ (see $\left.[11,29,30,31]\right)$ with the kernel

$$
\mathcal{N}=\operatorname{span}\left\{\sqrt{\mu}, \sqrt{\mu} v_{1}, \sqrt{\mu} v_{2}, \sqrt{\mu} v_{3}, \sqrt{\mu}|v|^{2}\right\} .
$$

From a rescaling argument (see Appendix 7.1), we can always assume that the initial condition $G$ satisfies

$$
G \in \mathcal{N}^{\perp} \text {. }
$$

In [30], For the radially symmetric case, the authors show that the linear Boltzmann operator behaves like the fractional harmonic oscillator $\mathcal{H}^{s}(0<s<1)$ with

$$
\mathcal{H}=-\Delta+\frac{|v|^{2}}{4}
$$

We study in the next section the spectral properties of the operators $\mathcal{L}$ and $\Gamma$.

2.2. The spectral problem. We introduce now an orthonormal basis of $L_{r}^{2}\left(\mathbb{R}^{3}\right)$ the radial symmetric functions of $L^{2}\left(\mathbb{R}^{3}\right)$ involving the generalized Laguerre polynomials $L_{n}^{\left[\ell+\frac{1}{2}\right]}$ : for that, we set for any $n \geq 0$

$$
\varphi_{n}(v)=\left(\frac{n !}{\sqrt{2} \Gamma(n+3 / 2)}\right)^{1 / 2} e^{-\frac{|v|^{2}}{4}} L_{n}^{\left[\frac{1}{2}\right]}\left(\frac{|v|^{2}}{2}\right) \frac{1}{\sqrt{4 \pi}}
$$

where $\Gamma(\cdot)$ is the standard gamma function, for all $x>0$,

$$
\Gamma(x)=\int_{0}^{+\infty} t^{x-1} e^{-x} d x
$$


and the Laguerre polynomial $L_{n}^{(\alpha)}$ of order $\alpha$, degree $n$ is

$$
L_{n}^{(\alpha)}(x)=\sum_{r=0}^{n}(-1)^{n-r} \frac{\Gamma(\alpha+n+1)}{r !(n-r) ! \Gamma(\alpha+n-r+1)} x^{n-r} .
$$

We have the spectral decomposition for the linear Boltzmann operator

$$
\mathcal{L} \varphi_{n}=\lambda_{n} \varphi_{n} \quad n \geq 0,
$$

with $\phi_{0}=\sqrt{\mu}, \lambda_{0}=0$ and for $n \geq 1$

$$
\lambda_{n}=2 \int_{0}^{\frac{\pi}{4}} \beta(\theta)\left(1-(\sin \theta)^{2 n}-(\cos \theta)^{2 n}\right) d \theta
$$

where $\beta(\theta)$ is defined from the collision kernel (see (1.2))

$$
\beta(\theta)=\sin \theta b(\cos \theta) \approx \frac{1}{|\theta|^{1+2 s}}, \quad 0<s<1, \theta \in\left(0, \frac{\pi}{2}\right] .
$$

The two families $\left(\varphi_{n}(v)\right)_{n \geq 0}$ and $\left(\lambda_{n}\right)_{n \geq 0}$ represent the eigenvectors and the eigenvalues of $\mathcal{L}$. Remark that this diagonalization of the linearized Boltzmann operator with Maxwellian molecules is also verified in the cutoff case (see [5, 11, 20, 29, 30]).

We consider the spectral expansion

$$
g(t, v)=\sum_{n=0}^{\infty} g_{n}(t) \varphi_{n}(v), \quad G(v)=\sum_{n=0}^{\infty} G_{n} \varphi_{n}(v)
$$

where $g_{n}(t)=\left(g(t, \cdot), \varphi_{n}(\cdot)\right)_{L^{2}}$ and $G_{n}=\left(G, \varphi_{n}\right)_{L^{2}}$. By definition, we have

$$
e^{-t \mathcal{L}} G(v)=\sum_{n=0}^{\infty} e^{-\lambda_{n} t} G_{n} \varphi_{n}(v)
$$

It is the solution of the equation

$$
\left\{\begin{array}{l}
\partial_{t} g^{\ell i n}+\mathcal{L} g^{\ell i n}=0 \\
g^{\ell i n}(0, v)=G(v)
\end{array}\right.
$$

Then the operator $\Gamma$ satisfies

$$
\Gamma\left(\varphi_{p}, \varphi_{q}\right)=\mu_{p q} \varphi_{p+q}
$$

where the non-linear eigenvalues are given by

$$
\mu_{p q}=\left(\frac{(2 p+2 q+1)}{(2 p+1)(2 q+1)} C_{2 p+2 q}^{2 p}\right)^{\frac{1}{2}} \int_{|\theta| \leq \frac{\pi}{4}} \beta(\theta)(\sin \theta)^{2 p}(\cos \theta)^{2 q} d \theta
$$

for $p \geq 1, q \geq 0$ and

$$
\mu_{0 q}=-\int_{|\theta| \leq \frac{\pi}{4}} \beta(\theta)\left(1-(\cos \theta)^{2 q}\right) d \theta
$$


for $q \geq 1$. Following [31], we therefore derive from (2.1) the following infinite system of ordinary differential equations :

$$
\left\{\begin{array}{l}
g_{0}^{\prime}(t)=0, \quad g_{1}^{\prime}(t)=0 \\
\text { for all } n \geq 2 \\
g_{n}^{\prime}(t)+\lambda_{n} g_{n}(t)=\sum_{\substack{p+q=n \\
0 \leq p, q \leq n}} \mu_{p q} g_{p}(t) g_{q}(t)
\end{array}\right.
$$

with the initial conditions (see (2.6))

$$
g_{n}(0)=G_{n} \text { for } \quad n \geq 0 .
$$

The goal is to study the behavior of each function $t \rightarrow g_{n}(t)$.

In the rest, we will focus on the computation and properties of this intermediate solution.

Proposition 2.1. We assume that $G \in \mathcal{N}^{\perp}$. Then the intermediate solution $h(t, v)$ defined by (2.2) satisfies

$$
h(t, v)=\sum_{n=0}^{\infty} h_{n}(t) \varphi_{n}(v)
$$

where $h_{0} \equiv h_{1} \equiv h_{2} \equiv h_{3} \equiv 0$ and for all $n \geq 4$

$$
h_{n}(t)=\sum_{\substack{p+q=n \\ 2 \leq p, q \leq n-2}} \int_{0}^{t} \mu_{p q} e^{-\left(\lambda_{p}+\lambda_{q}-\lambda_{n}\right) s}\left(G_{p}+h_{p}(s)\right)\left(G_{q}+h_{q}(s)\right) d s .
$$

Remark 2.2. As we have seen before, we divide the function $g$ in two parts as follows:

$$
g(t, v)=\underbrace{\sum_{n=0}^{\infty} e^{-\lambda_{n} t} G_{n} \varphi_{n}(v)}_{g^{\ell i n}(t, v)}+\underbrace{\sum_{n=0}^{\infty} e^{-\lambda_{n} t} h_{n}(t) \varphi_{n}(v)}_{g^{n \ell}(t, v)},
$$

therefore the formal solution $f(t, v)$ can be written as

$$
f(t, v)=\mu(v)+\sqrt{\mu(v)} \sum_{n=0}^{\infty}\left(e^{-\lambda_{n} t} G_{n}+e^{-\lambda_{n} t} h_{n}(t)\right) \varphi_{n}(v) .
$$

Proof of proposition 2.1. : As $G \in \mathcal{N}^{\perp}$, we get $G_{0}=G_{1}=0$ and we can verify from (2.8) that

$$
g_{0}(t)=g_{1}(t)=0, g_{2}(t)=G_{2} e^{-\lambda_{2} t}, g_{3}(t)=G_{3} e^{-\lambda_{2} t}
$$

and therefore $h_{0} \equiv h_{1} \equiv h_{2} \equiv h_{3} \equiv 0$. By (2.8), we may write

$$
g_{n}(t)=e^{-\lambda_{n} t} G_{n}+e^{-\lambda_{n} t} h_{n}(t)
$$

and

$$
g_{n}^{\prime}(t)+\lambda_{n} g_{n}(t)=\sum_{\substack{p+q=n \\ 2 \leq p, q \leq n-2}} \mu_{p q} g_{p}(t) g_{q}(t)
$$


We plug again the value of $g_{n}$ from (2.13) into the equation (2.14) and we get

$$
h_{n}^{\prime}(t)=e^{\lambda_{n} t} \sum_{\substack{p+q=n \\ 2 \leq p, q \leq n-2}} \mu_{p q} g_{p}(t) g_{q}(t) .
$$

Note that $h_{n}(0)=g_{n}^{n \ell}(0)=0$. Finally, plugging the expression of $g_{p}$ and $g_{q}$ from (2.13) into the previous equation and integrating we prove (2.10). Concerning the exact expression of the eigenvalue $\lambda_{n}$ and $\mu_{p q}$, see [31]. This concludes the proof.

We introduce now the following notations. For a $k$-uplet $\alpha \in \mathbb{N}^{k}$,

$$
\begin{gathered}
\Lambda_{\alpha}=\lambda_{\alpha_{1}}+\lambda_{\alpha_{2}}+\cdots+\lambda_{\alpha_{k}}, \\
G^{\alpha}=G_{\alpha_{1}} \times G_{\alpha_{2}} \cdots \times G_{\alpha_{k}} .
\end{gathered}
$$

Proposition 2.3. For each integer $n \geq 4$, we define $I_{n}$ a set of admissible indices

$$
I_{n}=\left\{\alpha \in \mathbb{N}^{k}\left|k \in \mathbb{N}^{*}, \alpha_{i} \geq 2,\right| \alpha \mid=n,\right\} .
$$

Then for each multi-index $\alpha, \beta, \in I_{n}$ there exists some real coefficients $c_{\beta}^{\alpha}$ which depends only on $\lambda_{2}, \ldots, \lambda_{n}$ and $\mu_{p q}$ for $2 \leq p, q \leq n-2$, $p+q \leq n$ such that

$$
h_{n}(t)=\sum_{\alpha, \beta \in I_{n}} c_{\beta}^{\alpha} G^{\alpha}\left(1-e^{-\left(\Lambda_{\beta}-\lambda_{n}\right) t}\right) .
$$

Proof. We compute directly from (2.10)

$$
h_{4}(t)=c_{(2,2)}^{(2,2)} G_{2}^{2}\left(1-e^{-\left(\Lambda_{(2,2)}-\lambda_{4}\right) t}\right)
$$

where

$$
c_{(2,2)}^{(2,2)}=\frac{\mu_{22}}{\left(\Lambda_{(2,2)}-\lambda_{4}\right)}
$$

and

$$
h_{5}(t)=c_{(2,3)}^{(2,3)} G_{2} G_{3}\left(1-e^{-\left(\Lambda_{(2,3)}-\lambda_{5}\right) t}\right)+c_{(3,2)}^{(3,2)} G_{3} G_{2}\left(1-e^{-\left(\Lambda_{(3,2)}-\lambda_{5}\right) t}\right)
$$

where

$$
c_{(2,3)}^{(2,3)}=\frac{\mu_{23}}{\left(\Lambda_{(2,3)}-\lambda_{5}\right)} \quad \text { and } \quad c_{(3,2)}^{(3,2)}=\frac{\mu_{32}}{\left(\Lambda_{(3,2)}-\lambda_{5}\right)} .
$$

We prove the result by induction. Then we can suppose that (2.15) is true for each $h_{n^{\prime}}\left(4 \leq n^{\prime} \leq n-1\right)$. We will use the integral expression (2.10) of $h_{n}$. We consider two integers $p, q$ such that $2 \leq p, q \leq n-2$ and $p+q=n$. Then from $(2.15)$

$$
\begin{aligned}
& h_{p}(t)=\sum_{\alpha, \beta \in I_{p}} c_{\beta}^{\alpha} G^{\alpha}\left(1-e^{-\left(\Lambda_{\beta}-\lambda_{p}\right) t}\right), \\
& h_{q}(t)=\sum_{\alpha^{\prime}, \beta^{\prime} \in I_{q}} c_{\beta^{\prime}}^{\alpha^{\prime}} G^{\alpha^{\prime}}\left(1-e^{-\left(\Lambda_{\beta^{\prime}}-\lambda_{q}\right) t}\right) .
\end{aligned}
$$


From the integral formula (2.10) we get

$$
h_{n}(t)=\int_{0}^{t} \sum_{\substack{p+q=n \\ 2 \leq p, q \leq n-2}}(A+B+C+D) d s
$$

with

$$
\begin{aligned}
& A=\mu_{p q} G_{p} G_{q} e^{-\left(\lambda_{p}+\lambda_{q}-\lambda_{n}\right) s}, \\
& B=\sum_{\alpha^{\prime}, \beta^{\prime} \in I_{q}} \mu_{p q} c_{\beta^{\prime}}^{\alpha^{\prime}} G_{p} G^{\alpha^{\prime}}\left(e^{-\left(\lambda_{p}+\lambda_{q}-\lambda_{n}\right) s}-e^{-\left(\lambda_{p}+\Lambda_{\beta^{\prime}}-\lambda_{n}\right) s}\right), \\
& C=\sum_{\alpha, \beta \in I_{p}} \mu_{p q} c_{\beta}^{\alpha} G^{\alpha} G_{q}\left(e^{-\left(\lambda_{p}+\lambda_{q}-\lambda_{n}\right) s}-e^{-\left(\Lambda_{\beta}+\lambda_{q}-\lambda_{n}\right) s}\right), \\
& D=\sum_{\alpha, \beta \in I_{p}} \sum_{\alpha^{\prime}, \beta^{\prime} \in I_{q}} \mu_{p q} c_{\beta}^{\alpha} c_{\beta^{\prime}}^{\alpha^{\prime}} G^{\alpha} G^{\alpha^{\prime}} \times \\
& \left(e^{-\left(\lambda_{p}+\lambda_{q}-\lambda_{n}\right) s}-e^{-\left(\Lambda_{\beta}+\lambda_{q}-\lambda_{n}\right) s}-e^{-\left(\lambda_{p}+\Lambda_{\beta^{\prime}}-\lambda_{n}\right) s}+e^{-\left(\Lambda_{\beta}+\Lambda_{\beta^{\prime}}-\lambda_{n}\right) s}\right) .
\end{aligned}
$$

Expanding each previous terms and integrating over $[0, t]$, we get the result (2.15) since each number $\lambda_{p}+\lambda_{q}-\lambda_{n}, \Lambda_{\beta}+\lambda_{q}-\lambda_{n}, \lambda_{p}+\Lambda_{\beta^{\prime}}-\lambda_{n}$, $\Lambda_{\beta}+\Lambda_{\beta^{\prime}}-\lambda_{n}$ are positive from the next lemma and $|\alpha|=|\beta|=p$, $\left|\alpha^{\prime}\right|=\left|\beta^{\prime}\right|=q$ and $p+q=n$.

Lemma 2.4. The linear eigenvalues $\lambda_{n}$ for the non-cutoff radially symmetric spatially homogeneous Boltzmann equation

$$
\lambda_{n}=\int_{|\theta| \leq \frac{\pi}{4}} \beta(\theta)\left(1-(\sin \theta)^{2 n}-(\cos \theta)^{2 n}\right) d \theta, \quad n \geq 2,
$$

verify the following property

$$
\lambda_{\alpha_{1}+\cdots+\alpha_{k}}<\lambda_{\alpha_{1}}+\cdots+\lambda_{\alpha_{k}}\left(=\Lambda_{\alpha}\right)
$$

for multi-index $\alpha \in(\mathbb{N} \backslash\{0,1\})^{k}$.

Proof. By [31], we may write

$$
\lambda_{\alpha_{1}+\alpha_{2}}<\lambda_{\alpha_{1}}+\lambda_{\alpha_{2}}
$$

then by iteration, we have

$$
\lambda_{\left(\alpha_{1}+\cdots+\alpha_{k}\right)+\alpha_{k+1}}<\lambda_{\alpha_{1}+\cdots+\alpha_{k}}+\lambda_{\alpha_{k+1}}<\left(\lambda_{\alpha_{1}}+\cdots+\lambda_{\alpha_{k}}\right)+\lambda_{\alpha_{k+1}} .
$$

\section{Numerical COMPUtations}

From now on, for sake of simplicity, we consider the specific case $s=\frac{1}{2}$ and

$$
\beta(\theta)=(\sin \theta)^{-2} .
$$

For the general case $s \in] 0,1[$ and other kernel $\beta$ which satisfies (2.5), we can compute some numerical approximations of the eigenvalues. We think that the results do not change. 
3.1. Computation of the eigenvalues. By the following assumption $\beta(\theta) \underset{0}{\approx} \frac{1}{|\theta|^{2}}$, we obtain (see [30])

$$
\lambda_{n} \underset{\infty}{\approx} \sqrt{n}
$$

where the linear eigenvalues $\lambda_{n}$ of $\mathcal{L}$ was defined in (2.4). We recall the value of $\lambda_{n}$ for $n \geq 2$ :

$$
\lambda_{n}=2 \int_{0}^{\frac{\pi}{4}} \beta(\theta)\left(1-(\sin \theta)^{2 n}-(\cos \theta)^{2 n}\right) d \theta .
$$

We compute the exact and approximate values of $\lambda_{n}$ by the following algorithm :

$$
\begin{aligned}
\lambda_{0} \leftarrow & 0 \\
\text { for } n & \text { from } 1 \text { to } N \text { do } \\
& \text { expr } \leftarrow \text { algebraic simplification of } \frac{1-\sin ^{2 n} \theta-\cos ^{2 n} \theta}{\sin ^{2} \theta} \\
& \lambda_{n}^{\text {exact }} \leftarrow \text { symbolic computation of } 2 \int_{0}^{\frac{\pi}{4}} \operatorname{expr} d \theta \\
& \lambda_{n}^{\text {approx }} \leftarrow \text { numerical computation of } \lambda_{n}^{\text {exact }}
\end{aligned}
$$

The "algebraic simplification" of "expr" removes the singularity when $\theta \rightarrow 0$ coming from the collision kernel $\beta(\theta)=\sin ^{-2} \theta$ (see (2.5)). It consists in a factorization of trigonometric polynomials. The symbolic computation of $\lambda_{n}^{\text {exact }}$ is reduced to compute the exact integral of a trigonometric polynomial. Then $\lambda_{n}^{\text {exact }}$ is approached numerically with a number of significant digits (equal to 10 in 1). The approximation is easily controlled by the estimate of the relative error $\left|\lambda_{n}^{\text {exact }}-\lambda_{n}^{\text {appr. }}\right| / \lambda_{n}^{\text {exact }}$. Using the software Maple ${ }^{\circledR} 13$, we finally get the numerical table 1.

\begin{tabular}{|c|c|c|c|}
\hline & Exact value & Approximate value & Relative error \\
\hline$\lambda_{1}$ & 0 & 0 & - \\
\hline$\lambda_{2}$ & $1+\frac{1}{2} \pi$ & 2.570796327 & $8.0 \times 10^{-11}$ \\
\hline$\lambda_{3}$ & $\frac{3}{2}+\frac{3}{4} \pi$ & 3.856194490 & $5.0 \times 10^{-11}$ \\
\hline$\lambda_{4}$ & $\frac{23}{12}+\frac{15}{16} \pi$ & 4.861909780 & $1.2 \times 10^{-10}$ \\
\hline$\lambda_{5}$ & $\frac{55}{24}+\frac{35}{32} \pi$ & 5.727783632 & $8.2 \times 10^{-11}$ \\
\hline$\lambda_{10}$ & $\frac{61717}{16128}+\frac{109395}{65536} \pi$ & 9.070756042 & $9.0 \times 10^{-11}$ \\
\hline$\lambda_{15}$ & $\frac{41349267}{8200192}+\frac{35102025}{16777216} \pi$ & 11.61545300 & $3.2 \times 10^{-10}$ \\
\hline$\lambda_{20}$ & $\frac{60225247403}{9906683904}+\frac{83945001525}{34359738368} \pi$ & 13.75454524 & $2.5 \times 10^{-11}$ \\
\hline
\end{tabular}

TABLE 1. Symbolic and numerical computation of $\lambda_{n}$. 
The approximation of eigenvalues can be controlled to be sufficiently precise for upcoming computations. For a general kernel $\beta(\theta)$, there is in general no more explicit values. But some classical numerical methods can be easily applied. Nevertheless, there is no more any algebraic simplification, and it is necessary to treat carefully the singularity.

3.2. Computation of the nonlinear eigenvalues. We recall the coefficients $\mu_{p q}$ from (2.7): for some $p, q \geq 1$

$$
\mu_{p q}=\sqrt{\frac{(2 p+2 q+1)}{(2 p+1)(2 q+1)} C_{2 p+2 q}^{2 p}} \int_{|\theta| \leq \frac{\pi}{4}} \beta(\theta)(\sin \theta)^{2 p}(\cos \theta)^{2 q} d \theta .
$$

We compute the exact and the approximate value of $\mu_{p q}$ (again with a relative error $\approx 10^{-10}$ ) for $1 \leq p+q \leq N$ by the following algorithm :

$$
\begin{aligned}
& \text { for } p \text { from } 1 \text { to } N \text { do } \\
& \text { for } q \text { from } 0 \text { to } N-p d o \\
& \quad \text { expr } \leftarrow \text { symbolic computation of } 2 \int_{0}^{\frac{\pi}{4}} \sin ^{2 p-2} \theta \cos ^{2 q} \theta d \theta \\
& \quad \mu_{p q}^{\text {exact }} \leftarrow \sqrt{\frac{(2 p+2 q+1)}{(2 p+1)(2 q+1)} C_{2 p+2 q}^{2 p}} \times \text { expr } \\
& \mu_{p q}^{\text {approx }} \leftarrow \text { numerical computation of } \mu_{p q}^{\text {exact }}
\end{aligned}
$$

We present in the table 2 of results for $p+q=n=2, \ldots, 5,20$. The sin-

\begin{tabular}{|c|c|c|c|c|}
\hline$n=2$ & $n=3$ & $n=4$ & $n=5$ & $n=20$ \\
\hline$\mu_{1,1} \approx 2.35$ & $\mu_{1,2} \approx 2.88$ & $\mu_{1,3} \approx 3.29$ & $\mu_{1,4} \approx 3.62$ & $\mu_{1,19} \approx 6.68$ \\
\hline & $\mu_{2,1} \approx 0.519$ & $\mu_{2,2} \approx 0.702$ & $\mu_{2,3} \approx 0.84$ & $\mu_{2,18} \approx 1.55$ \\
\hline & & $\mu_{3,1} \approx 0.196$ & $\mu_{3,2} \approx 0.30$ & $\mu_{3,17} \approx 0.75$ \\
\hline & & & $\mu_{4,1} \approx 0.084$ & $\mu_{4,16} \approx 0.46$ \\
\hline & & & & $\vdots$ \\
\hline & & & & $\mu_{19,1} \approx 10^{-5}$ \\
\hline
\end{tabular}

TABLE 2. Numerical computation of $\mu_{p q}$.

gularity coming from the collision kernel $\beta(\theta)=\sin ^{-2} \theta$ is removed by a simple simplification (remark the exponent $(2 p-2)$ of the sinus term of $\left.\mu_{p q}\right)$. Again for a general collision kernel, the values of these nonlinear eigenvalues can be approximated by classical numerical methods. 
3.3. Numerical solutions of the linear problem. We introduce from (2.11) the approximation of the linear solution

$$
g_{N}^{\ell i n}(t, v)=\sum_{n=0}^{N} e^{-\lambda_{n} t} G_{n} \varphi_{n}(v)
$$

where the reals $G_{n}$ are the given initial spectral coefficients. In order to compute the value of the linear solution, we use the formula (2.3) of the eigenfunction $\varphi_{n}$ which involves the generalized Laguerre polynomials $L_{n}^{\left[\ell+\frac{1}{2}\right]}$. We get the following algorithm :

for $n$ from 0 to $\mathrm{N}$ do

$$
\varphi_{n}(v) \leftarrow\left(\frac{n !}{\sqrt{2} \Gamma(n+3 / 2)}\right)^{1 / 2} e^{-\frac{|v|^{2}}{4}} L_{n}^{\left[\frac{1}{2}\right]}\left(\frac{|v|^{2}}{2}\right) \frac{1}{\sqrt{4 \pi}}
$$

Finally, we obtain the linear solution by the sum :

$$
\begin{aligned}
& g_{N}^{\ell i n}(t, v) \leftarrow 0 \\
& \text { for } n \text { from } 2 \text { to } N \text { do } \\
& \qquad g_{N}^{\ell \text { lin }}(t, v) \leftarrow g_{N}^{\ell \text { in }}(t, v)+e^{-\lambda_{n} t} G_{n} \varphi_{n}(v)
\end{aligned}
$$

We estimate the $L^{2}$ theoretical error $\left(g^{\ell i n}-g_{N}^{\ell i n}\right)$ for the different initial data $G$ used for computation in the next sections.

Proposition 3.1. We consider the solution of the following linear problem

$$
\left\{\begin{array}{l}
\partial_{t} g^{\ell i n}+\mathcal{L} g^{\ell i n}=0 \\
g^{\ell i n}(0, v)=G(v)
\end{array}\right.
$$

We have the following estimates :

1) For initial data $G \in L^{2}$,

$$
\left\|g^{\ell i n}(t, \cdot)-g_{N}^{\ell i n}(t, \cdot)\right\|_{L^{2}} \lesssim e^{-c \sqrt{N} t}\|G\|_{L^{2}} .
$$

2) For the measure initial data $G$ defined by (5.1) (see also proposition 7.3), there exist some constants $C>0$ and $c>0$ such that for $t>0$

$$
\left\|g^{\ell i n}(t, \cdot)-g_{N}^{\ell i n}(t, \cdot)\right\|_{L^{2}} \lesssim \frac{1}{t^{b}} e^{-\gamma \sqrt{N} t} .
$$

Proof. The solution of (3.3) is

$$
g^{\ell i n}(t, v)=\sum_{n=0}^{\infty} e^{-\lambda_{n} t} G_{n} \varphi_{n}(v) .
$$

The exact error in $L^{2}$ is

$$
\left\|g^{\ell i n}(t, \cdot)-g_{N}^{\ell i n}(t, \cdot)\right\|_{L^{2}}^{2}=\sum_{n=N+1}^{\infty} e^{-2 \lambda_{n} t}\left|G_{n}\right|^{2} .
$$

1) If $G \in L^{2}\left(\mathbb{R}_{v}^{3}\right)$, then as we have from (3.1)

$$
\left\|g^{\ell i n}(t, \cdot)-g_{N}^{\ell i n}(t, \cdot)\right\|_{L^{2}}^{2}=\sum_{n=N+1}^{\infty} e^{-2 \lambda_{n} t}\left|G_{n}\right|^{2} \lesssim e^{-2 c \sqrt{N} t}\|G\|_{L^{2}}^{2}
$$


We can deduce that the exact error tends to zero when $N$ tends to infinity.

2) We suppose now that $\mathrm{F}$ is the measure initial data $\mu+\delta$. We can approximate the spectral coefficients $G_{n}$ of $\mathrm{G}$ by $n^{\frac{1}{4}}$ and by 3.1 we can then find some positive constants $c$ and $C$ such that

$$
\left\|g^{\ell i n}(t, \cdot)-g_{N}^{\ell i n}(t, \cdot)\right\|_{L^{2}}^{2} \leq C \sum_{n=N+1}^{\infty} e^{-c \sqrt{n} t} n^{2} .
$$

We consider the function $\rho_{t}$ defined on $\mathbb{R}_{+}$by $\rho_{t}(x)=e^{-c \sqrt{x} t} x^{2}$. So that $\rho_{t}$ is positive, continuous and decreasing for $x \geq 16 /(c t)^{2}$, therefore by using the Cauchy integral criterion, we can write the following inequality :

$$
\left\|g^{\ell i n}(t, \cdot)-g_{N}^{\ell i n}(t, \cdot)\right\|_{L^{2}} \leq \frac{C}{t^{b}} e^{-\gamma \sqrt{N} t} \underset{N \rightarrow \infty}{\longrightarrow} 0
$$

where $b$ and $\gamma$ are some positive constants.

3.4. Numerical solutions of the non-linear part. Concerning the nonlinear part $g^{n \ell}=e^{-t \mathcal{L}} h$ of the solution, we consider the partial series

$$
g_{N}^{n \ell}(t, v)=\sum_{n=0}^{N} e^{-\lambda_{n} t} h_{n}(t) \varphi_{n}(v) .
$$

We then use the decomposition of $h$ in the spectral basis (2.9) and the integral formula (2.10) to compute $h_{n}(t)$. Therefore we solve explicitly the system (2.8) by the following algorithm:

$$
\begin{aligned}
& h_{0}(t), h_{1}(t), h_{2}(t), h_{3}(t) \leftarrow 0 \\
& \text { for } n \text { from } 4 \text { to } N \text { do } \\
& \quad S \leftarrow 0 \\
& \text { for } p \text { from } 4 \text { to } n d o \\
& \quad q \leftarrow n-p \\
& \quad S \leftarrow S+\mu_{p q}\left(G_{p}+h_{p}(t)\right)\left(G_{q}+h_{q}(t)\right) e^{-\left(\lambda_{p}+\lambda_{q}-\lambda_{n}\right) t} \\
& \quad h_{n}(t) \leftarrow \text { symbolic computation of } \int_{0}^{t} S
\end{aligned}
$$

The exact computation of the integral $\int_{0}^{t} S$ is straightforward since, from proposition 2.3, the symbolic expression $S$ is an linear combination of exponential terms $e^{\alpha t}$. We get the exact following solutions of the system of integral formula (2.10):

$$
\begin{aligned}
& h_{0}=h_{1}=h_{2}=h_{3}=0, \\
& h_{4}=\frac{\mu_{22}}{\lambda_{2}+\lambda_{2}-\lambda_{4}} G_{2}^{2}\left(1-e^{-\left(\lambda_{2}+\lambda_{2}-\lambda_{4}\right) t}\right), \\
& h_{5}=\frac{\mu_{23}+\mu_{32}}{\lambda_{2}+\lambda_{3}-\lambda_{5}} G_{2} G_{3}\left(1-e^{-\left(\lambda_{2}+\lambda_{3}-\lambda_{5}\right) t}\right),
\end{aligned}
$$


From the symbolic expression of $h_{n}$ we compute the numerical approximation :

$$
\begin{aligned}
h_{0}= & h_{1}=h_{2}=h_{3}=0, \\
h_{4}= & 2.51 G_{2}^{2}\left(1-e^{-0.279 t}\right), \\
h_{5}= & 1.62 G_{2} G_{3}\left(1-e^{-0.698 t}\right), \\
h_{6}= & 0.322 G_{3}^{2}\left(1-e^{-1.20 t}\right)+1.17\left(1-e^{-0.928 t}\right) G_{2} G_{4} \\
& +\left(-2.95 e^{-0.928 t}+0.677+2.26 e^{-1.20 t}\right) G_{2}^{3}, \\
h_{7}= & 0.501 G_{2} G_{5}\left(1-e^{-1.09 t}\right)+0.220\left(1-e^{-1.51 t}\right) G_{3} G_{4} \\
& +\left(0.201+0.478 e^{-1.79 t}-0.274 e^{-1.51 t}-0.407 e^{-1.09 t}\right) G_{2}^{2} G_{3}, \\
& \ldots
\end{aligned}
$$

We finally get from (3.4) the approximation $g_{N}^{n \ell}$ of the nonlinear part of the solution $g^{n \ell}(t, v)$ by the following algorithm :

$$
\begin{aligned}
& g_{N}^{n \ell}(t, v) \leftarrow 0 \\
& \text { for } n \text { from } 2 \text { to } N \text { do } \\
& \qquad g_{N}^{n \ell}(t, v) \leftarrow g_{N}^{n \ell}(t, v)+e^{-\lambda_{n} t} h_{n}(t) \varphi_{n}(v)
\end{aligned}
$$

The symbolic and numerical computation of the nonlinear part of the solution plays the main difficulty of our method. We analyze the computation time and rounding off error in the next section.

3.5. Discussions on the symbolic computation. From the computation of the linear (3.2) and nonlinear (3.4) part, we calculate the approximated solution of the Boltzmann equation (1.1)

$$
f_{N}=\mu+\sqrt{\mu}\left(g_{N}^{\ell i n}+g_{N}^{n \ell}\right) .
$$

The method using the software Maple ${ }^{\circledR} 13$ and its internal function "int $(f(x), x=a . . b)$ " for symbolic computation of integrals seems limited to a number $N$ around 20, since for $N=20$, the number of terms of $h_{20}$ is around 5000 and the computation time is around 50 seconds. Moreover they are both exponentially increasing (see Figure 1). We now estimate the truncation and rounding error due to the software computations. For a regular $L^{2}$ initial data we have computed the solution $f_{N}$ for $N=20$ and different number of digits (we can control the number of digits that Maple ${ }^{\circledR} 13$ uses when making calculations with software floating-point numbers). We set $P_{1}$ and $P_{2}$ two numbers of digits and we compare the two numerical solutions $f_{N}^{P_{1}}$ and $f_{N}^{P_{2}}$ computed respectively using $P_{1}$ and $P_{2}$. We define the rounding relative error as

$$
\text { error }=\frac{\left\|f_{N}^{P_{1}}-f_{N}^{P_{2}}\right\|_{\infty}}{\left\|f_{N}^{P_{2}}\right\|_{\infty}}
$$

and we get the following results for different choices of $\left(P_{1}, P_{2}\right)$ : We check from the table 3 that the relative error is roughly 10 times the 
(a) Complexity

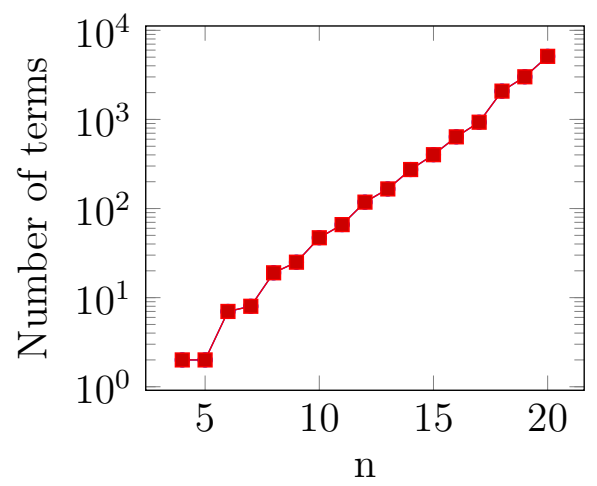

(b) Computation time

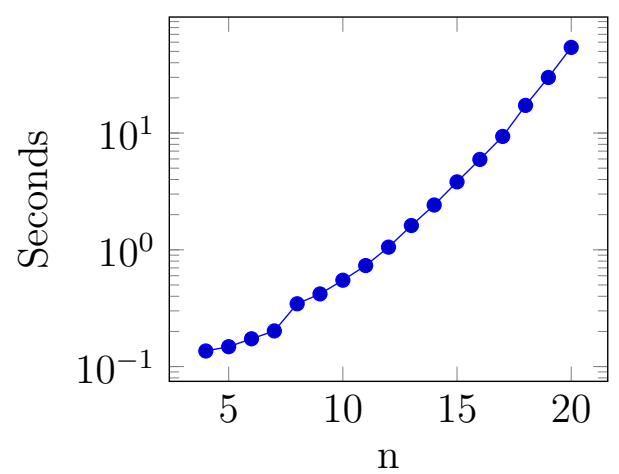

Figure 1. Number of terms for $h_{n}$ and computation time in seconds for $h_{1}, \ldots, h_{n}$.

\begin{tabular}{|c|c|c|c|c|c|}
\hline$\left(P_{1}, P_{2}\right)$ & $(10,20)$ & $(20,30)$ & $(30,40)$ & $(40,50)$ & $(50,100)$ \\
\hline error & $3.810^{-4}$ & $2.310^{-15}$ & $2.010^{-29}$ & $8.210^{-40}$ & $1.410^{-49}$ \\
\hline
\end{tabular}

TABLE 3. Relative rounding off error.

precision of the computation of $f_{N}^{P_{1}}$. The figure 2 represents the computation time of the solution $f_{N}$ with a regular $L^{2}$ initial data and for different numbers of digits. The computations of the solution $f_{N}$ was

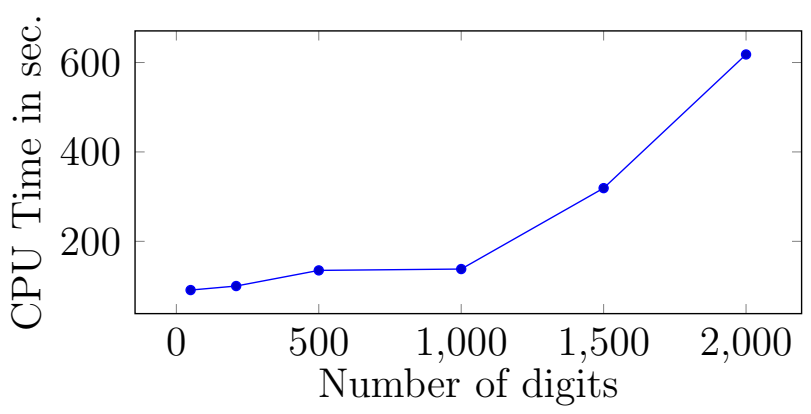

Figure 2. Computation time for different number of digits.

run on a computer having 8 Xeon processors $2.33 \mathrm{GHz}$ with $8 \mathrm{~GB}$ of memory. The method using Maple ${ }^{\circledR} 13$ on this computer seems limited to a number around $N=20$. Surprisingly, the computation time is roughly the same (around 90 seconds) for a number of digits between 20 and 1000. The main part of this time is therefore used for algebraic manipulation.

We present in the two upcoming sections the results of the computation for different initial values. 


\section{Radial Bi-Gaussian initial VAlue}

We set the initial data :

$$
\tilde{F}(w)=\frac{1}{(2 \pi)^{\frac{3}{2}}}\left(\exp \left(-\frac{1}{2}(|w|+1)^{2}\right)+\exp \left(-\frac{1}{2}(|w|-1)^{2}\right)\right) .
$$

We next rescale the initial data following lemma 7.1. We show in figure 3 the spectral approximation $F_{N}(v)$ of the initial data $F(v)$ such that $F_{N}(v)=\mu(v)+\sqrt{\mu(v)} G_{N}(v)$ where $G_{N}(v)=\sum_{n=0}^{N} G_{n} \varphi_{n}(v)$. We then compute the solutions $h_{n}(t)$ from the proposition 2.1 for $n=$

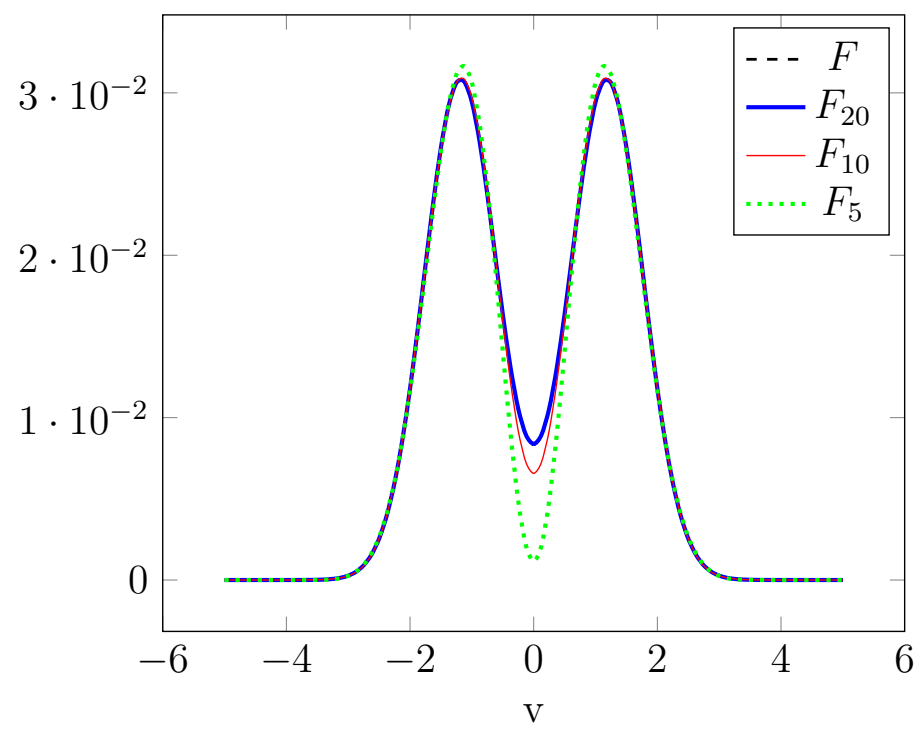

FiguRE 3. Approximation of the initial data

$4,5, \ldots, N$ with $N=20$. For each integer $n$, the function $t \rightarrow h_{n}(t)$ is monotone and tends to a finite limit when $t$ tends to infinity (See Figure $4(\mathrm{a}))$. We recall that $h_{n}(t)$ is a finite sum of decreasing exponential terms (see section 3.4). Since the initial data $G$ is a regular function, the spectral coefficients $G_{n}$ are exponentially decreasing. The numerical computation of $h_{n}(t)$ shows also that $\left\|h_{n}\right\|_{\infty}$ is exponentially decreasing with respect to $n$ (See Figure $4(\mathrm{~b})$ ). In this special case, the linear part $e^{-t \mathcal{L}} G$ and the nonlinear part $e^{-t \mathcal{L}} h$ have roughly the same behavior. We present in figure 5 the graph of the linear part and nonlinear part and the ratio in $L^{2}$-norm

$$
R_{N}(t)=\frac{\left\|g_{N}^{n \ell}(t, \cdot)\right\|_{L^{2}}}{\left\|g_{N}^{\ell i n}(t, \cdot)\right\|_{L^{2}}}=\frac{\left(\sum_{n=4}^{N}\left|e^{-\lambda_{n} t} h_{n}(t)\right|^{2}\right)^{\frac{1}{2}}}{\left(\sum_{n=2}^{N}\left|e^{-\lambda_{n} t} G_{n}\right|^{2}\right)^{\frac{1}{2}}}
$$

We observe that the nonlinear part is very small compared to the other. We remark that in this case the series (3.2) and (3.4) behave as 
(a) $t \mapsto h_{n}(t)$

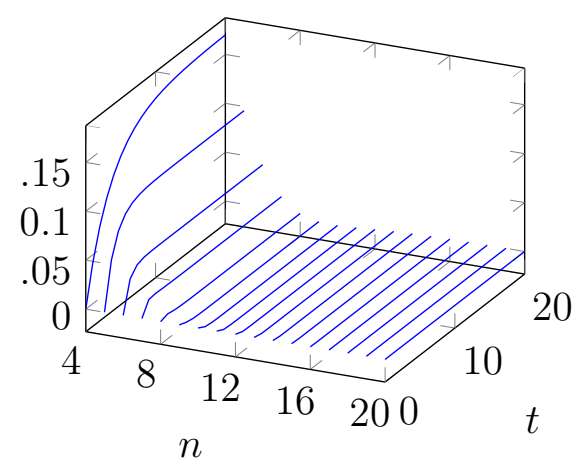

(b) $\frac{\sup _{t}\left|h_{n}(t)\right|}{\left|G_{n}\right|}$

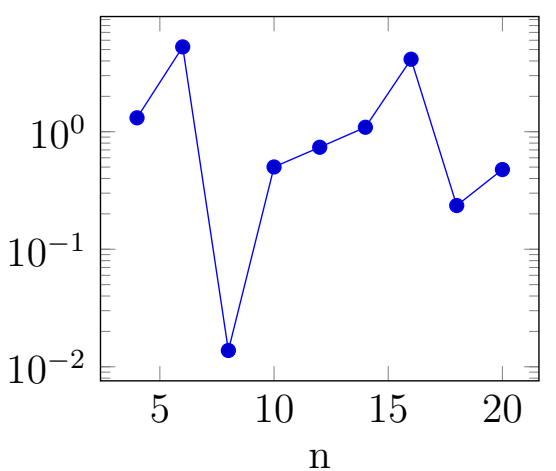

Figure 4. Behavior of the nonlinear part $h_{n}$.
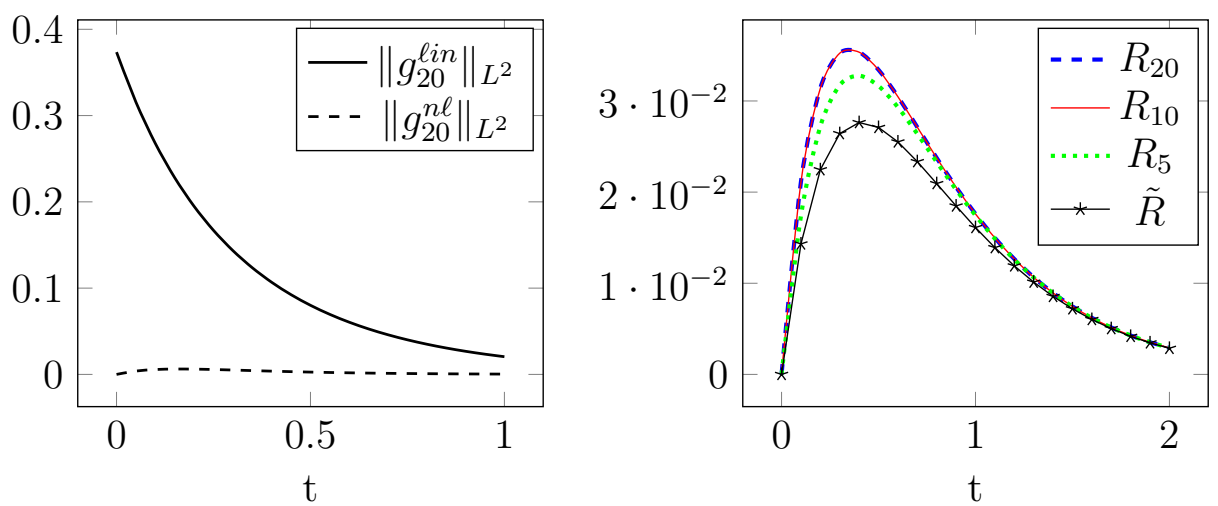

Figure 5. Comparison of the non linear part with respect to the linear part (see (3.2), (3.4), (4.1), (4.2)).

$$
\begin{aligned}
& g_{N}^{\ell i n}(t, v) \approx e^{-\lambda_{2} t} G_{2} \varphi_{2}(v), \\
& g_{N}^{n \ell}(t, v) \approx 2.51 e^{-\lambda_{4} t} G_{2}^{2}\left(1-e^{-\left(2 \lambda_{2}-\lambda_{4}\right) t}\right) \varphi_{4}(v),
\end{aligned}
$$

because the terms of $h_{n}(t)$ are composed of products of terms which are numerically converging to zero. The quotient (for $G_{2} \neq 0$ ) of the two previous approximations behaves closely like the ratio (see (4.1))

$$
R_{N}(t) \approx \tilde{R}(t) \stackrel{\text { def }}{=} e^{-\left(\lambda_{4}-\lambda_{2}\right) t}\left|G_{2}\right|\left(1-e^{-\left(2 \lambda_{2}-\lambda_{4}\right) t}\right) .
$$

We finaly compute (see figure 6) the solution $f=\mu+\sqrt{\mu} g$ using the spectral Hermite eigenfunctions $\varphi_{n}(v)$ and the expansion (2.12) of $g$ in this basis. Since the function $g(t, \cdot) \in \mathcal{N}^{\perp}$ for all time $t \geq 0$, the approximate solution $f_{N}(t, \cdot)$ is naturally orthogonal to $\varphi_{0}$ and $\varphi_{1}$. Therefore is a conservation of the mass and the energy. Finally, we check that the approximate solution $f_{N}$ converges to the Gaussian function when the time tends to infinity. 


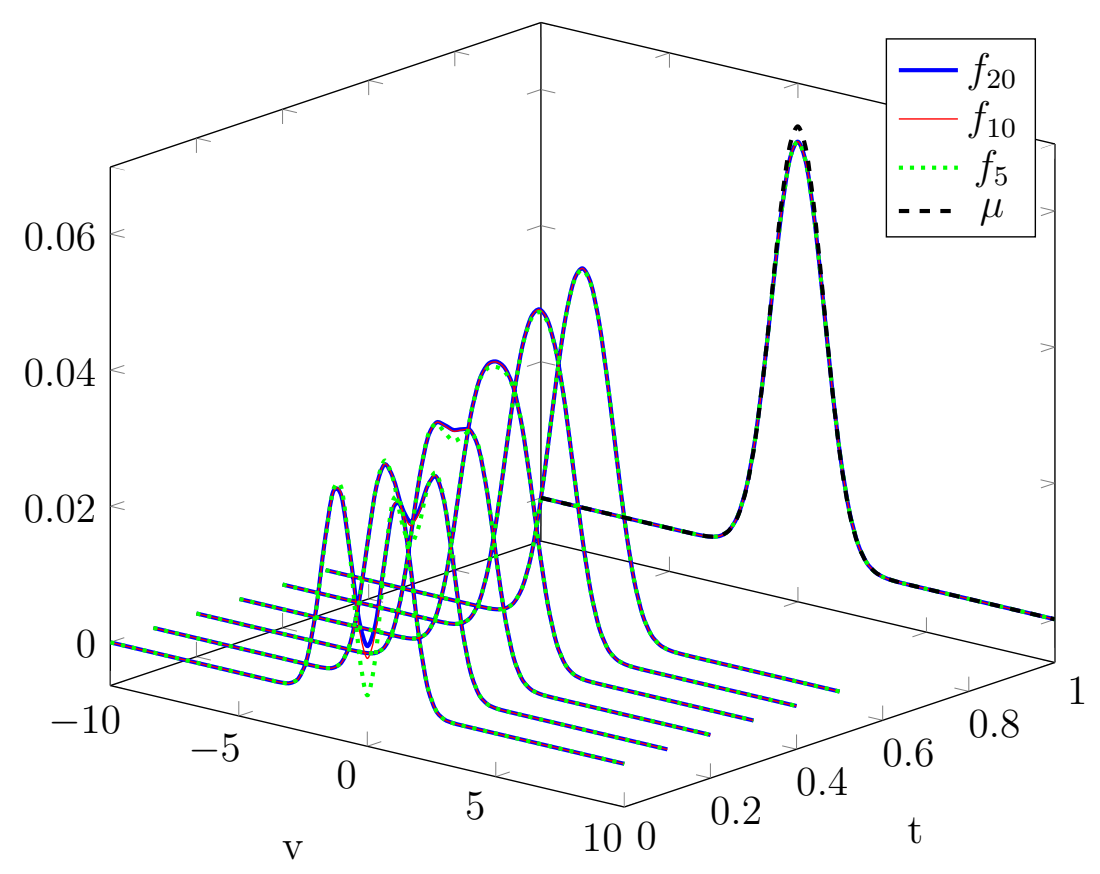

Figure 6. Graph of $(t, v) \mapsto f_{N}(t, v)$ for $N=5,20$ and $\mu(v)$.

\section{Numerical RESUlts FOR INITIAL MEASURE DATA}

We consider the initial measure data

$$
\tilde{F}=\text { gaussian }+ \text { Dirac }=\mu+\delta .
$$

Following the lemma and rescaling the solution $F(v)=2^{-\frac{5}{2}} \tilde{F}\left(2^{-\frac{1}{2}} v\right)$, we get the normalized initial data

$$
\left\{\begin{array}{l}
F(v)=2^{-\frac{5}{2}} \mu\left(2^{-\frac{1}{2}} v\right)+2^{-1} \delta(v), \\
G(v)=2^{-\frac{13}{4}} \pi^{-\frac{3}{4}}-\sqrt{\mu(v)}+2^{-\frac{1}{4}} \pi^{\frac{3}{4}} \delta(v) .
\end{array}\right.
$$

We verify that $\left\langle G, \varphi_{0}\right\rangle=\left\langle G, \varphi_{1}\right\rangle=0$ and therefore $G \in \mathcal{N}^{\perp}$. We then compute the spectral coefficients for $n \geq 0$ (see proposition 7.1):

$$
G_{n}=\left\langle G, \varphi_{n}\right\rangle=\frac{1+(-1)^{n}}{2}\left(\frac{(2 n+1) !}{2^{2 n}(n !)^{2}}\right)^{\frac{1}{2}} .
$$

Note that the coefficients $G_{2 n+1}$ are equal to zero and we have the following approximation of $G$ :

$$
G(v) \approx \sum_{n=1}^{\infty} n^{\frac{1}{4}} \varphi_{2 n}(v) .
$$

We set $F_{\text {reg }}(v)=2^{-\frac{5}{2}} \mu\left(2^{-\frac{1}{2}} v\right)$ the regular part of the distribution $F$. We check in the left figure 7 that the approximate initial data behaves as a Dirac function. 

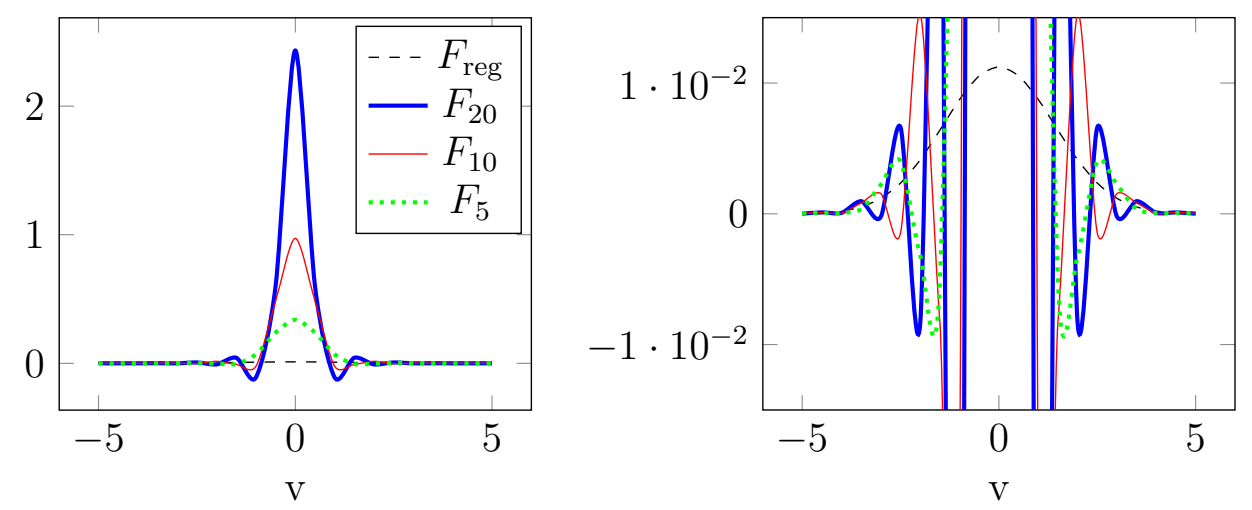

FiguRE 7. Approximation of the initial data.

Remark that to capture the approximation of the regular part $F_{\text {reg }}$, we have to rescale the cote $y$-coordinate. We observe the oscillations of $F_{N}$ which are expected since the functions $F_{N}$ approach the Dirac function when $N$ tends to infinity (see the right figure 7 ). We now focus on the evolution problem. As the initial data is a distribution, we can check that the linear part of the solution is singular :

$$
\left\|g^{\ell i n}(t, \cdot)\right\|_{L^{2}}^{2}=\sum_{n=2}^{\infty} G_{n}^{2} e^{-2 \lambda_{n} t} \approx \frac{1}{t^{\alpha}}, \quad \text { when } t \rightarrow 0
$$

for some $\alpha>0$ (since $G_{2 n} \approx n^{\frac{1}{4}}$ and $\left.\lambda_{n} \approx n^{\frac{1}{2}}\right)$. We next compute the nonlinear part $h_{n}(t)$ of the solution (see the left figure 8 ).

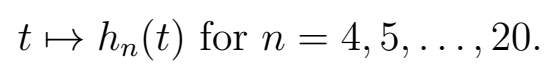

$$
\frac{\sup _{t}\left|h_{n}(t)\right|}{\left|G_{n}\right|} \text { for } n=4,6, \ldots, 20 .
$$
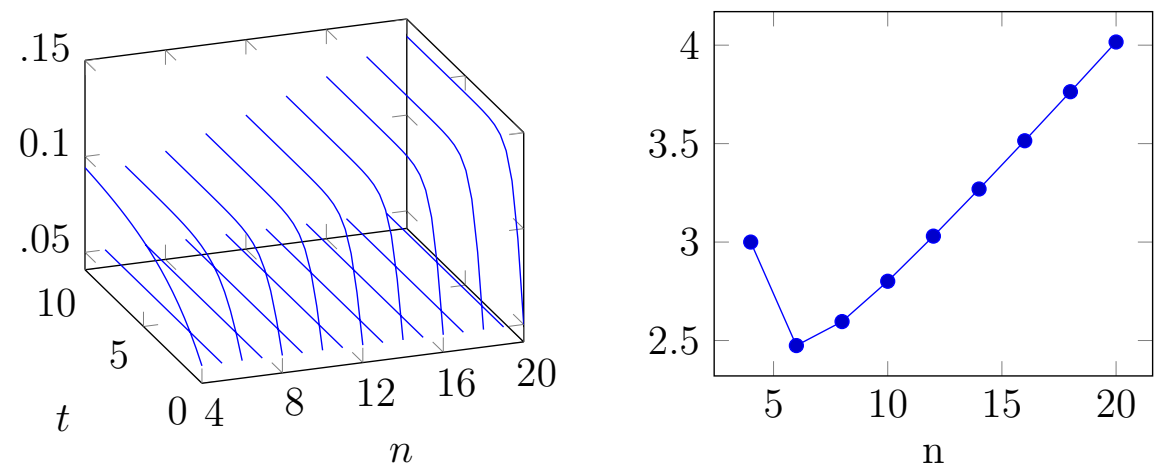

FiguRE 8. Behavior of the nonlinear part $h_{n}$.

We observe some numerical evidences that these functions are increasing less than a power of $n$ :

$$
\sup _{t \geq 0}\left|h_{n}(t)\right| \leq C n^{a}
$$


with $a$ close to 1 . Since $G_{n} \approx n^{\frac{1}{4}}$, the behavior of a term of the series $\left(g_{N}^{\ell i n}(t)+g_{N}^{n \ell}(t)\right)$ is dominated by the nonlinear part.

We next calculate the linear part and nonlinear part of solution (see Figure 9). The numerical computations in the left figure show that
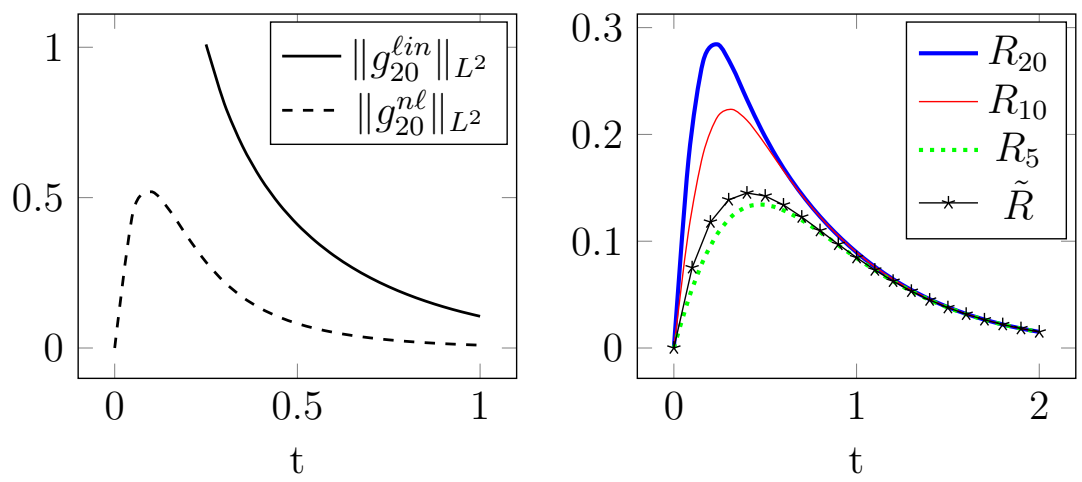

Figure 9. Comparaison of the non linear part with respect to the linear part (see (3.2), (3.4), (4.1), (4.2)).

$g_{N}^{n \ell}(t)$ is a regular function for all time and we verify also that $g_{N}^{\ell i n}(t)$ is singular as $t \rightarrow 0$ as pointed in (5.2). For a large time, the $L^{2}$ norm of the linear part $\left(\sim e^{-\lambda_{2} t}\right)$ dominates the norm of the nonlinear part $\left(\sim e^{-\lambda_{4} t}\right)$, and the ration $R_{N}(t)$ has the same behavior as in the previous section (see (4.2)).

We then compute the numerical approximation $f_{N}$ of the solution $f$ for $N=20$ and we check that the solution behaves as a Dirac function as $t \rightarrow 0$ and tends to the Gaussian as $t \rightarrow \infty$ (see Figure 10).

Since $\lambda_{n} \approx c \sqrt{n}$ and if the behavior of $\sup _{t \geq 0}\left|h_{n}(t)\right|$ is dominated by a power of $n$ (which is numerically verified), then we have for some $b, \gamma>0$ :

$$
\forall t>0,\left\|f_{N}(t, \cdot)-f(t, \cdot)\right\|_{L^{2}} \lesssim \frac{1}{t^{b}} e^{-\gamma \sqrt{N} t} \rightarrow 0 \quad \text { as } \quad N \rightarrow \infty .
$$

We observe some other numerical evidences that the series converges in $L^{2}$ for $t>0$ and the solution converges to a Gaussian as $t \rightarrow \infty$.

\section{Conclusion}

We have considered the perturbation $g$ of the solution $f$ of the Boltzmann equation defined by

$$
f=\mu+\sqrt{\mu} g \quad \text { where } \quad g(t, v)=\sum_{n} g_{n}(t) \varphi_{n}(v)
$$

and we have studied the behavior of the spectral coefficients

$$
g_{n}(t)=e^{-\lambda_{n} t}\left(G_{n}+h_{n}(t)\right), \quad g_{n}(0)=G_{n} .
$$




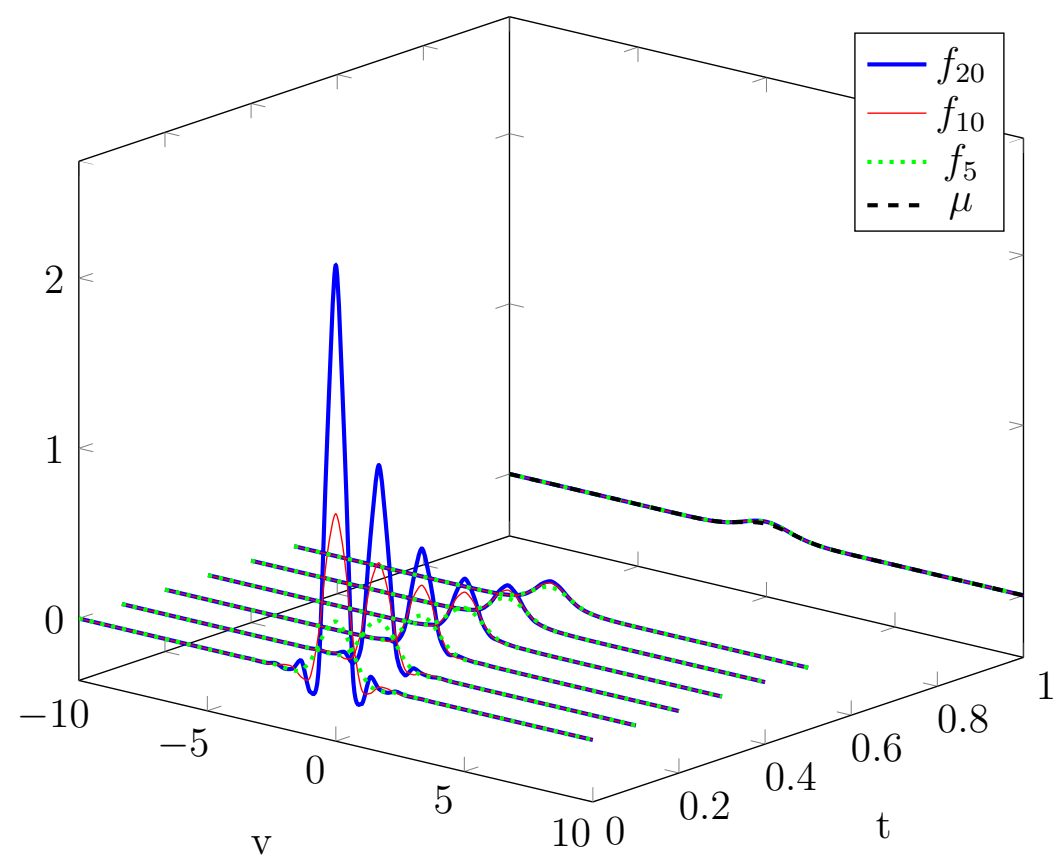

Figure 10. Graph of $(t, v) \mapsto f_{N}(t, v)$ for $N=5,10$, 20 and $\mu(v)$.

We have then computed formally the spectral coefficients $h_{n}(t)$ for $n=$ $0,1, \ldots, N$ with $N=20$. We have checked also the results for small $L^{2}$ initial data and distribution type initial data $\mu+\delta$.

- For small $L^{2}$ initial data, our method was tested with several $L^{2}$ initial conditions : $\mathrm{F}$ is a sum of two Gaussian, $G_{n}=\frac{0.1}{n}, G_{n}=\frac{1}{n}$. The results show that there are some numerical evidences that the spectral series $\sum_{n} e^{-\lambda_{n} t}\left(G_{n}+h_{n}(t)\right) \varphi_{n}$ is convergent in $L^{2}$ for any time $t \geq 0$ and the solution converges to a Gaussian. Moreover, for large times, the linear part $G_{n}$ is preponderant with respect to the non-linear part $h_{n}(t)$.

- For the distribution type initial data $\mu+\delta$, the simulations show some numerical evidences that the spectral series converges in $L^{2}$ and there is a regularization of the solution for $t>0$.

We have computed the formal solutions of the spectral coefficients $h_{n}$ of the solution of the Boltzmann equation. If there exists a regular solution for $t>0$, then the solutions $h_{n}$ are the exact projections of the solution on the spectral basis. These calculations were made in the case of a non-cutoff kernel. The numerical results are coherent for small $L^{2}$ initial data or for the distribution case $\mu+\delta$. There is conservation of the mass, momentum and energy of the approximated solution (since $g_{N}(t, \cdot)$ is orthogonal to the kernel $\mathcal{N}$ for all time). Moreover the approximated solution $f_{N}(t, \cdot)$ (defined in (3.5)) converges to a Gaussian when $t$ tends to infinity. 


\section{ACKNOWLEDGMEnTs}

The authors wish to thank Chao-Jiang Xu for interesting discussions.

\section{APPENDIX}

7.1. Rescaling of the solution. We consider a radial solution $\tilde{f}(s, w)$ of the Boltzmann equation :

$$
\left\{\begin{array}{l}
\partial_{s} \tilde{f}=\mathbf{Q}(\tilde{f}, \tilde{f}) \\
\left.\tilde{f}\right|_{t=0}=\tilde{F}
\end{array}\right.
$$

Lemma 7.1. We consider the functions $f(t, v)$ and $F(v)$ defined by the change of variable

$$
\left\{\begin{array}{l}
f(t, v)=\alpha \tilde{f}\left(\frac{\alpha}{\beta^{3}} t, \beta v\right) \\
F(v)=\alpha \tilde{F}(\beta v)
\end{array}\right.
$$

where

$$
\alpha=\frac{\left(\frac{1}{3} \int_{\mathbb{R}^{3}} w^{2} \tilde{F}(w) d w\right)^{\frac{3}{2}}}{\left(\int_{\mathbb{R}^{3}} \tilde{F}(w) d w\right)^{\frac{5}{2}}} \text { and } \beta=\frac{\left(\frac{1}{3} \int_{\mathbb{R}^{3}} w^{2} \tilde{F}(w) d w\right)^{\frac{1}{2}}}{\left(\int_{\mathbb{R}^{3}} \tilde{F}(w) d w\right)^{\frac{1}{2}}} .
$$

Therefore $f(t, v)$ is a solution of the Boltzmann equation (1.1) with initial data F. Moreover, if we set $F=\mu+\sqrt{\mu} G$, we then have $G \in \mathcal{N}^{\perp}$.

Remark 7.2. If $F$ is such that

$$
\begin{aligned}
& \int_{\mathbb{R}^{3}} F(v) d v=\int_{\mathbb{R}^{3}} \mu(v) d v=1, \\
& \int_{\mathbb{R}^{3}} v^{2} F(v) d v=\int_{\mathbb{R}^{3}} v^{2} \mu(v) d v=3,
\end{aligned}
$$

then the function $G$ defined by $G=\frac{1}{\sqrt{\mu}}(F-\mu)$ belongs to $\mathcal{N}^{\perp}$.

Proof. It is easy to check that $f(t, v)$ is a solution of the Boltzmann equation. Since $G$ is a radial function, it is enough to check that

$$
(G, \sqrt{\mu})_{L^{2}}=\left(G,|v|^{2} \sqrt{\mu}\right)_{L^{2}}=0 .
$$

Recalling that $\left(\varphi_{p}, \varphi_{q}\right)_{L^{2}}=\delta_{p q}$,

$$
\varphi_{0}=\sqrt{\mu} \quad \text { and } \quad \varphi_{1}=6^{-\frac{1}{2}}\left(3-|v|^{2}\right) \sqrt{\mu},
$$

it is equivalent to prove

$$
\left(F / \sqrt{\mu}, \varphi_{0}\right)_{L^{2}}=1 \quad \text { and } \quad\left(F / \sqrt{\mu}, \varphi_{1}\right)_{L^{2}}=0,
$$

which gives the equations

$$
\left\{\begin{array}{l}
\int_{\mathbb{R}^{3}} F(v) d v=\int_{\mathbb{R}^{3}} \mu(v) d v=1 \\
\int_{\mathbb{R}^{3}}|v|^{2} F(v) d v=\int_{\mathbb{R}^{3}}|v|^{2} \mu(v) d v=3 .
\end{array}\right.
$$


Using the change of variable $w=\beta v$, we can check that if we set the values of $\alpha$ and $\beta$ given in the lemma, the previous equations are fulfilled.

7.2. Measure initial data. We define the following distribution initial data :

$$
\tilde{F}=\mu+\delta \text {. }
$$

Following the rescaling of lemma 7.1, we compute

$$
\begin{aligned}
\langle\tilde{F}, 1\rangle & =\int_{\mathbb{R}^{3}} \mu(v) 1 d v+\langle\delta, 1\rangle=2, \\
\left\langle\tilde{F}, v^{2}\right\rangle & =\frac{1}{3} \int_{\mathbb{R}^{3}} \mu(v) v^{2} d v+\left\langle\delta, v^{2}\right\rangle=1
\end{aligned}
$$

and then $\alpha=2^{-\frac{5}{2}}$ and $\beta=2^{-\frac{1}{2}}$. Using the change of variable $w=\beta v$, we get the new rescaled distribution initial data

$$
F=\alpha \tilde{F} \circ(\beta \text { Id })=2^{-\frac{5}{2}}\left(\mu\left(2^{-\frac{1}{2}} \cdot\right)+\left(2^{\frac{1}{2}}\right)^{3} \delta\right) .
$$

Proposition 7.3. We consider the initial data

$$
F=2^{-\frac{5}{2}}\left(\mu\left(2^{-\frac{1}{2}} \cdot\right)+\left(2^{\frac{1}{2}}\right)^{3} \delta\right)
$$

and we set $G$ such that $F=\mu+\sqrt{\mu} G$. Then we have

$$
G=-\sqrt{\mu}+2^{-\frac{13}{4}} \pi^{-\frac{3}{4}}+2^{-\frac{1}{4}} \pi^{\frac{3}{4}} \delta .
$$

We consider the coordinates $G_{n}=\left\langle G, \varphi_{n}\right\rangle$ of the distribution $G$ in the spectral basis $\left(\varphi_{n}\right)_{n}$. We can check that

$$
G_{0}=G_{1}=0
$$

and for all integer $n \geq 2$,

$$
G_{n}=\left\langle G, \varphi_{n}\right\rangle=\frac{1+(-1)^{n}}{2}\left(\frac{(2 n+1) !}{2^{2 n}(n !)^{2}}\right)^{\frac{1}{2}} .
$$

Proof. The expression of $G$ follows from the definition of the Gaussian $\mu$. We then compute

$$
\left\langle G, \varphi_{n}\right\rangle=-\left(\varphi_{0}, \varphi_{n}\right)_{L^{2}}+2^{-\frac{13}{4}} \pi^{-\frac{3}{4}}\left(1, \varphi_{n}\right)_{L^{2}}+2^{-\frac{1}{4}} \pi^{\frac{3}{4}} \varphi_{n}(0)
$$

and the conclusion results directly from lemma 7.5.

We consider now a special Gaussian approximation $F_{\varepsilon} \in L^{2}$ of the distribution initial data $F=\mu+\delta$ and we obtain some spectral stability result in this case.

Proposition 7.4. We consider the initial data for $\varepsilon>0$

$$
\tilde{F}_{\varepsilon}(w)=\mu(w)+\frac{1}{\varepsilon^{3}} \mu\left(\frac{w}{\varepsilon}\right) .
$$


Following lemma \%.1, the rescaled initial data of $\tilde{F}_{\varepsilon}$ is $F_{\varepsilon}=\mu+\sqrt{\mu} G_{\varepsilon}$ where $G_{\varepsilon} \in \mathcal{N}^{\perp}$ and

$$
G_{\varepsilon}(v)=-\sqrt{\mu(v)}+2^{-\frac{5}{2}}\left(1+\varepsilon^{2}\right)^{3 / 2}\left(\sqrt{\mu(\varepsilon v)}+\frac{1}{\varepsilon^{3}} \sqrt{\mu(v / \varepsilon)}\right) .
$$

Then we have the following limit in the sense of distribution as $\varepsilon \rightarrow 0$ :

$$
\begin{aligned}
& F_{\varepsilon} \rightarrow F=2^{-\frac{5}{2}}\left(\mu\left(2^{-\frac{1}{2}} \cdot\right)+\left(2^{\frac{1}{2}}\right)^{3} \delta\right), \\
& G_{\varepsilon} \rightarrow G=-\sqrt{\mu}+2^{-\frac{13}{4}} \pi^{-\frac{3}{4}}+2^{-\frac{1}{4}} \pi^{\frac{3}{4}} \delta .
\end{aligned}
$$

The coordinates of $G_{\varepsilon}$ in the spectral basis $\left(\varphi_{n}\right)_{n \geq 0}$ are given by:

$$
\begin{aligned}
G_{\varepsilon, 0} & =G_{\varepsilon, 1}=0, \\
G_{\varepsilon, n} & =\frac{1+(-1)^{n}}{2} \frac{\left(1-\varepsilon^{2}\right)^{n}}{\left(1+\varepsilon^{2}\right)^{n}}\left(\frac{(2 n+1) !}{2^{2 n}(n !)^{2}}\right)^{\frac{1}{2}}, \quad \forall n \geq 2 .
\end{aligned}
$$

Moreover we have $G_{\varepsilon, n} \rightarrow G_{n}$ as $\varepsilon$ tends to 0 as $\varepsilon \rightarrow 0$ where $G_{n}$ is given in (7.1).

Remark. There is continuity of the spectral coefficients : $G_{\varepsilon, n} \rightarrow G_{n}$ as $\varepsilon$ tends to 0 .

Proof. From Lemma 7.1, we set

$$
F_{\varepsilon}(v)=\alpha_{\varepsilon} \tilde{F}_{\varepsilon}\left(\beta_{\varepsilon} v\right)
$$

where

$$
\begin{aligned}
& \alpha_{\varepsilon}=\frac{\left(\frac{1}{3} \int_{\mathbb{R}^{3}} w^{2} \tilde{F}_{\varepsilon}(w) d w\right)^{\frac{3}{2}}}{\left(\int_{\mathbb{R}^{3}} \tilde{F}_{\varepsilon}(w) d w\right)^{\frac{5}{2}}}=\frac{\sqrt{2}}{8}\left(1+\varepsilon^{2}\right)^{3 / 2}, \\
& \beta_{\varepsilon}=\frac{\left(\frac{1}{3} \int_{\mathbb{R}^{3}} w^{2} \tilde{F}_{\varepsilon}(w) d w\right)^{\frac{1}{2}}}{\left(\int_{\mathbb{R}^{3}} \tilde{F}_{\varepsilon}(w) d w\right)^{\frac{1}{2}}}=\frac{\sqrt{2}}{2}\left(1+\varepsilon^{2}\right)^{1 / 2} .
\end{aligned}
$$

Then $F_{\varepsilon}=\mu+\sqrt{\mu} G_{\varepsilon}$ where

$$
G_{\varepsilon}(v)=-\sqrt{\mu(v)}+\frac{2^{\frac{3}{4}}\left(1+\varepsilon^{2}\right)^{3 / 2}}{16 \pi^{3 / 4}}\left(e^{-\frac{\varepsilon^{2} v^{2}}{4}}+\frac{1}{\varepsilon^{3}} e^{-\frac{v^{2}}{4 \varepsilon^{2}}}\right)
$$

or

$$
G_{\varepsilon}(v)=-\sqrt{\mu(v)}+\frac{2^{\frac{1}{2}}\left(1+\varepsilon^{2}\right)^{3 / 2}}{8}\left(\sqrt{\mu(\varepsilon v)}+\frac{1}{\varepsilon^{3}} \sqrt{\mu(v / \varepsilon)}\right),
$$

then $G_{\varepsilon} \in \mathcal{N}^{\perp}$. We compute from lemma 7.5

$$
G_{\varepsilon, n}=\left(G_{\varepsilon}, \varphi_{n}\right)_{L^{2}}=I_{1}+\frac{2^{\frac{1}{2}}\left(1+\varepsilon^{2}\right)^{3 / 2}}{8}\left(I_{2}+I_{3}\right)
$$


where

$$
\begin{aligned}
& I_{1}=\left(-\sqrt{\mu}, \varphi_{n}\right)_{L^{2}}=\left(-\varphi_{0}, \varphi_{n}\right)_{L^{2}}=-\delta_{0, n}, \\
& I_{2}=\left(\sqrt{\mu(\varepsilon \cdot)}, \varphi_{n}\right)_{L^{2}}=\left(2^{\frac{9}{4}} \pi^{\frac{3}{4}}\right) \varphi_{n}(0) \frac{\left(1-\varepsilon^{2}\right)^{n}}{\left(1+\varepsilon^{2}\right)^{n+3 / 2}}, \\
& I_{3}=(-1)^{n} I_{2} .
\end{aligned}
$$

Finally we get :

$$
G_{\varepsilon, n}=-\delta_{0, n}+\frac{1+(-1)^{n}}{2} \frac{\left(1-\varepsilon^{2}\right)^{n}}{\left(1+\varepsilon^{2}\right)^{n}}\left(\frac{(2 n+1) !}{2^{2 n}(n !)^{2}}\right)^{\frac{1}{2}} .
$$

7.3. Some results on the spherical harmonics. We recall that

$$
\varphi_{n}(v)=\left(\frac{n !}{\sqrt{2} \Gamma(n+3 / 2)}\right)^{1 / 2} e^{-\frac{|v|^{2}}{4}} L_{n}^{\left[\frac{1}{2}\right]}\left(\frac{|v|^{2}}{2}\right) \frac{1}{\sqrt{4 \pi}}
$$

where the Laguerre polynomial $L_{n}^{(\alpha)}$ of order $\alpha$, degree $n$ is

$$
L_{n}^{(\alpha)}(x)=\sum_{r=0}^{n}(-1)^{n-r} \frac{\Gamma(\alpha+n+1)}{r !(n-r) ! \Gamma(\alpha+n-r+1)} x^{n-r} .
$$

Lemma 7.5. For $a>0$ and $n \geq 0$ we have

$$
\begin{aligned}
& \varphi_{n}(0)=\frac{1}{(2 \pi)^{\frac{3}{4}}}\left(\frac{(2 n+1) !}{2^{2 n}(n !)^{2}}\right)^{\frac{1}{2}} \\
& \int_{\mathbb{R}^{3}} \varphi_{n}(v) d v=(-1)^{n} 2^{3} \pi^{\frac{3}{2}} \varphi_{n}(0), \\
& \left(\sqrt{\mu(a \cdot)}, \varphi_{n}\right)_{L^{2}}=\left(2^{\frac{9}{4}} \pi^{\frac{3}{4}}\right) \varphi_{n}(0) \frac{\left(1-a^{2}\right)^{n}}{\left(1+a^{2}\right)^{n+3 / 2}} .
\end{aligned}
$$

Proof. These equalities come from classical properties of the Hermite funtions (we have checked them using Maple ${ }^{\circledR} 13$ for integers $n \leq 20$ ).

\section{REFERENCES}

[1] R. Alexandre, Y. Morimoto, S. Ukai, C.-J. Xu, T. Yang, Regularizing Effect and Local Existence for the Non-Cutoff Boltzmann Equation, Arch Rational Mech Anal, 198, 39-123, 2010.

[2] M. Bennoune, M. Lemou, L. Mieussens, Uniformly stable numerical schemes for the Boltzmann equation preserving the compressible Navier-Stokes asymptotics, J. Comp. Phys. 227, 3781-3803, 2008.

[3] G. Bird, Molecular gas dynamics and the direct simulation of gas flows, Oxford University Press, 2nd Edition 1994.

[4] A. Bobylev, Exact solutions of the Boltzmann equation, In Akademiia Nauk SSSR Doklady, 225, 1296-1299, 1975.

[5] A. Bobylev, The theory of the nonlinear spatially uniform Boltzmann equation for Maxwell molecules, Soviet Sci. Rev. Sect. C: Math. Phys., 7, 111-233, 1988. 
[6] A. Bobylev, A. Palczewski, J. Schneider, On approximation of the Boltzmann equation by discrete velocity models, Comptes rendus de l'Académie des sciences. Série 1, Mathématique, 320(5), 639-644, 1995.

[7] A. Bobylev, S. Rjasanow, Difference scheme for the Boltzmann equation based on fast fourier transform, Technical report 1996.

[8] L. Boltzmann, Weitere studien über das wärmegleichgewicht unter gas-molekülen, Wiener Berichte, 66, 275-370, 1872.

[9] C. Buet, S. Cordier, P. Degond, Regularized Boltzmann operators, Simulation methods in kinetic theory, Comput. Math. Appl., 35, 55-74, 1998.

[10] Z. Cai, Y. Fan, L. Ying, Entropy monotonic spectral method for Boltzmann equation. arXiv:1704.07369v1 [math.NA] 23 Apr 2017.

[11] C. Cercignani, The Boltzmann equation and its applications, Applied Mathematical Sciences, Springer-Verlag, New York, 67, 1988.

[12] L. Desvillettes, Regularization Properties of the 2-Dimensional Non Radially Symmetric Non Cutoff Spatially Homogeneous Boltzmann Equation for Maxwellian Molecules, Transport Theory and Statistical Physics, vol. 26, n. 3, 341-357, 1997.

[13] L. Desvillettes, Regularization for the non Cutoff 2D Radially Symmetric Boltzmann Equation with a Velocity Dependant Cross Section, Transport Theory and Statistical Physics, vol. 25, n. 3-5, 383-394, 1996 (special issue).

[14] L. Desvillettes, F. Golse, On the Smoothing Properties of a Model Boltzmann Equation without Grad's Cutoff Assumption, Proceedings of the 21st International Symposium on Rarefied Gas Dynamics, R. Brun, R. Campargue, R. Gatignol et J.-C. Lengrand éditeurs, vol. 1, 47-54, Cépaduès éditions, Toulouse 1999.

[15] L. Desvillettes, F. Golse, On a Model Boltzmann Equation without Angular Cutoff, Differential and Integral Equations, vol. 13, n. 4-6, 567-594, 2000.

[16] L. Desvillettes, B. Wennberg, Smoothness of the Solution of the Spatially Homogeneous Boltzmann Equation without Cutoff, Communications in Partial Differential Equations, vol. 29, n.1 et 2 , 133-155, 2004.

[17] G. Dimarco, L. Pareschi, High order asymptotic-preserving schemes for the Boltzmann equation, C. R. Math. Acad. Sci. Paris 350 , 9-10, 481-486, 2012.

[18] G. Dimarco, L. Pareschi, Asymptotic preserving implicit-explicit Runge-Kutta methods for nonlinear kinetic equations, SIAM J. Numer. Anal. 51, 2, 10641087, 2013.

[19] G. Dimarco, L. Pareschi, Numerical methods for kinetic equations, Acta Numerica, Cambridge University Press (CUP), 23, 369-520, 2014.

[20] E. Dolera, On the spectrum of the linearized Boltzmann collision operator for Maxwellian molecules, Boll. UMI, 46, 67-105, 2010.

[21] F. Filbet, C. Mouhot, L. Pareschi, Solving the Boltzmann equation in N log2 N, SIAM J. Sci. Comput. 28, 3, 1029-1053, 2007.

[22] I.M. Gamba; J.R. Haack, C.D.Hauck and J.Hu, A fast spectral method for the Boltzmann collision operator with general collision kernels, SIAM J. for Scientific Computing, 2017, to appear.

[23] D Goldstein, B Sturtevant, JE Broadwell, Investigations of the motion of discrete-velocity gases, Progress in Astronautics and Aeronautics, 117, 100$117,1989$.

[24] P.-T. Gressman, R.-M. Strain, Global Classical Solutions of the Boltzmann equation without angular cut-off, Journal of the American Mathematical Society, 24 (3), 771-847, 2011.

[25] S. Jin, Runge-Kutta methods for hyperbolic conservation laws with stiff relaxation terms, J. Comp. Phys. 122, 51-67, 1995. 
[26] S. Jin, Efficient asymptotic-preserving (ap) schemes for some multiscale kinetic equations, SIAM J. Sci. Comput. 21, 84, 441-454, 1999.

[27] S. Jin, Asymptotic preserving (AP) schemes for multiscale kinetic and hyperbolic equations, a review, Riv. Math. Univ. Parma (N.S.) 3 , 2, 177-216, 2012.

[28] M. Krook and T.T. Wu, Exact solutions of the Boltzmann equation, The Physics of Fluids, 20(10), 1589-1595, 1977.

[29] N. Lerner, Y. Morimoto, K. Pravda-Starov, C.-J. Xu, Phase space analysis and functional calculus for the linearized Landau and Boltzmann operators, Kinet. Relat. Models 6, 625-648, 2013.

[30] N. Lerner, Y. Morimoto, K. Pravda-Starov, C.-J. Xu, Spectral and phase space analysis of the linearized non-cutoff Kac collision operator, J. Math. Pures Appl. 100, 832-867, 2013.

[31] N. Lerner, Y. Morimoto, K. Pravda-Starov, C.-J. Xu, Gelfand-Shilov smoothing properties of the radially symmetric spatially homogeneous Boltzmann equation without angular cutoff, J. Differential Equations, 256, 797-831, 2014.

[32] C. Mouhot, L. Pareschi, Fast algorithms for computing the Boltzmann collision operator, Math. Comp., 75(256), 1833-1852, 2006.

[33] V.A. Panferov, A.G. Heintz, A new consistent discrete-velocity model for the Boltzmann equation, Mathematical Methods in the Applied Sciences, 25(7), 571-593, 2002.

[34] L. Pareschi, B. Perthame, A Fourier spectral method for homogeneous Boltzmann equations, Transport Theory and Statistical Physics, 25(3-5), 369-382, 1996.

[35] L. Pareschi, G. Russo, Numerical solution of the Boltzmann equation I: Spectrally accurate approximation of the collision operator, SIAM J. Numer. Anal. $37,4,1217-1245,2000$.

[36] F. Rogier, J. Schneider, A direct method for solving the Boltzmann equation, Transport Theory and Statistical Physics, 23(1-3), 313-338, 1994.

[37] C. Villani, On a new class of weak solutions to the spatially homogeneous Boltzmann and Landau equations, Arch. Rational Mech. Anal., 143, 273-307, 1998.

[38] C. Villani, A review of mathematical topics in collisional kinetic theory, Handbook of mathematical fluid dynamics, 1, 71-74, 2002.

E-mail address: leo.glangetas@univ-rouen.fr

E-mail address: brahimjrad92@hotmail.com 